\title{
On Some Linguistic Peculiarities of Internet Slang
}

\author{
Narineh Madoyan \\ Yerevan State University
}

T $\mathrm{n}$ examining language in social context we come to see that the ongoing social processes do have great impact on the evolution of language. Language itself can't be taken apart from society so the changes in the latter will subsequently affect the language, i.e. language is the reflection of the social situation and the shifts in social situation result in implementing new means of communication.

In a given culture or subgroup, common ground, such as mutual knowledge, mutual beliefs, and mutual expectations, shift to accommodate the circumstances and the parties with whom we are conversing. The widespread use of the Internet forces the members of the virtual community to create and establish new linguistic means which enable them to communicate within the scope of the virtual medium. As a result a new type of communication has emerged and become an inseparable part of our reality. As there is no unanimously accepted term for this new discourse variety, it is most generally referred to as Internet language or Internet discourse. Many alternative terms, such as: "Netlish", "Weblish", "Cyberspeak", "Computer-Mediated Communication (CMC), "Electronic Language"/“E-language", "Online English", "Virtual Discourse”, etc. are also widely used. The famous British linguist and founder of Internet Linguistics David Crystal prefers the term "Netspeak" to "Netlish" or "Weblish" on the grounds that the latter two are associated with English which is not exactly the language used on the Net, while "Netspeak" inherently reflects the multilingual character of the Internet discourse (Crystal 2004:17). Another term to define the aforementioned variety of speech is "Internet slang", which, although in the broad sense of the word refers to the Internet language in general, in the narrow sense of the word is somehow associated with the notion that the word "slang" expresses, i.e. informal language that is more common in speech than in writing and is typically restricted to a particular context or group (http://www.wordreference.com).

It is the distinctive features of the "narrow sense" Internet slang that is investigated in the present paper.

Slang is an informal set of words and phrases that are used to reinforce or establish one's identity within a social group or a trend in society (Eble 1996:11). Slang is an important aspect of language to touch on because it is vocabulary that embodies the social functions of language. Slang seems to be as old as language itself given that it is part of ordinary interactions in all languages in which communities are large and diverse enough to have identifiable subgroups (Eble 1996:11).

Based on the existent theoretical approaches Internet slang goes far beyond the general descriptions of slang in general. But as studies show Internet slang as an existing linguistic phenomenon shares some global characteristics common to slang. Internet slang is the broad definition to acronyms ${ }^{1}$, abbreviations ${ }^{2}$ and emoticons ${ }^{3}$ created to eliminate keystrokes online and distinguish the newly emerged discourse and its users. 
According to Bethany K. Dumas and Jonathan Lighter, an expression should be considered "true slang" if it meets at least two of the following criteria:

- It lowers, if temporarily, the dignity of formal or serious speech or writing.

- Its use implies that the user is familiar with whatever is referred to, or with a group of people that are familiar with it and use the term.

- It is a taboo term in ordinary discourse with people of higher social status or greater responsibility.

- It replaces "a well-known conventional synonym". This is done primarily to avoid "the discomfort caused by the conversational item or by further elaboration" (Dumas, Lighter 1953:5-17).

People well-aware of conventions of Netspeak will obviously agree that the slang used on the Net meets even more than two of the above mentioned criteria.

Internet slang consists of slang that users of the Internet have developed and utilized and as compared with general slang it does belong to the subculture of the virtual medium. As mentioned above many terms of the Internet slang originated with the purpose of saving keystrokes. The following examples are obvious illustrations of the notion: $u$ for "you", $r$ for "are", 4 for "for", etc. The slang terms often appear in lower case, with capitals they are reserved for emphatic use. For example, the first person singular pronoun "I" is mainly used as $i$, but when a certain amount of attention is needed, the capitalized version is used.

Those who by no means are involved in the subculture of the virtual community may find this type of code language unintelligible. Sometimes users make up Internet abbreviations on the spot, therefore many of them seem ambiguous, vague, or even nonsensical to those who are far beyond the scope of the culture of virtual medium.

Due to the widespread use of the Internet today, more and more new words are being coined to denote newly originated computer technicalities, leet being one of them. Also known as eleet, the term denotes an alternative alphabet for the English language that is primarily on the Internet. Leet is traditionally used by programmers and online gamers, while much larger communities of Internet users commonly use Internet slang.

The vocabulary of the Internet users has really come to convey some innovative characteristics of slang. One of the main obstacles of the virtual communication is that the interactants don't have the possibility to express their emotions and thoughts in the way they do in face-to-face communication. Unlike in verbal communication, where paralinguistic features are a key factor for the communicators to express clearly their true emotive state of the moment, in virtual communication the speakers are deprived of expressing happiness, fear, love, anger through voice, facial expression and gestures. In such cases Netizens may use emoticons to express their feelings. They may utilize emoticons both genuinely and sarcastically; for example $-P$ as an emoticon expresses either genuine amusement and a sense of fun, or a negative sarcastic comment on something another user might have said.

The range of emoticons coined to express feelings may be infinite and can't be limited as any individual is capable of devising something new and denoting an own meaning. As studies show, Netizens seem to have developed many slang terms over the years. 
Acronyms are an inseparable part of the Internet slang. Acronyms have always been an integral part of computer culture, and they have since spawned a new language on the Internet. One of the main characteristics of acronyms is that you pronounce the letters as a new word. For example, $F U B A R$ (fouled up beyond all recognition) is pronounced foo-bar and $R A D A R$ (respecting accuracy in domestic abuse reporting) is pronounced ray-dar. Chat acronyms or chaq (pronounced chalk) originally developed on pre-Internet bulletin board systems. It should also be noted that acronyms are generally typed in all capital letters.

As far as emoticons are concerned there seems to exist a subdivision according to which emoticons can be classified into Western and Eastern types. The Western use of emoticons is much different than the Eastern use. Western style emoticons are frequently written from left to right. Generally, a colon is used for the eyes of the face. The combination of a colon with other graphic symbols may designate different emotive states:

- : ) shows smiley or happy face ( (),

- :D is used for a big smile,

- : - D is used to express laughter,

- D - : is used to indicate madness

- : :/:/ expresses annoyance,

- : - ( is used to express frown (<http://www.netlingo.com $>$ ).

In contrast to Western codes for smiley faces, eastern emoticons can be read without tilting the head. Easterners focus much greater attention on the eyes and make significantly more errors than the Westerners. The cultural specificity in eye movements that they show is probably a reflection of cultural specificity in facial expression. Westerners use the whole face to convey emotion while Easterners use the eyes more and mouth less. We can assume that culture not only has a great impact on the verbal but also on the virtual communication. primarily use the mouth to convey emotional states, e.g. :) for happy and : ( for sad, whereas use the eyes, e.g. $\wedge . \wedge$ for happy and; ; for sad (<http://www.netlingo.com $>)$.

The pragmatic aspect of the Internet language may also be a source of interesting revelations. Being devoid of any paralinguistic features, Internet language may give rise to many misinterpretations. Internet writing is, by its nature difficult to interpret, especially in chat rooms, because much of it is quickly input and many assume falsely their interlocutors know their "body" language. In order to be able to convey one's true communicative goal, it is best to be as explicit as possible in the process of the virtual communication. Members of the virtual community must be cautious with the choice and arrangement of graphic symbols, as the misplacement of the same symbol may cause misunderstanding between communicators. The arrangement of the symbols must be precise as, for example, the colon combined with bracket may express both positive and negative emotions, a colon combined with a dash and an opening bracket is used to express dissatisfaction, while the combination of the latter with closing bracket expresses good mood or happiness.

As mentioned before, one of the important identifying characteristics of slang is its group-identifying function. Slang is often used when the user wants to be accepted by a select social group (Eble 1996:119). A strong sense of belonging can stem from the shar- 
ing and maintaining of an ever-changing vocabulary that undoubtedly serves to include and exclude members from social groups (Eble 1996:119). The same tendencies are noticeable within many groups of Internet users, especially the chatgroups. ${ }^{4}$ Howard Rheingold describes the Internet as an "ecosysytem" of subcultures (Rhiengold 1993:3). Crystal states that the Internet users want to interact only with those who share their common interests, i.e. belong to the same subculture (Crystal 2004:60). This means that the Internet situation imposes some constraints on the use of linguistic means by the members of the virtual community. Crystal enumerates some identifiable subgroups among which he enlists hackers who elaborated their own slang and which enables them to distinguish between hackers and non-hackers, thus identifying one as an insider and the other as an outsider (Crystal 2004:67). Most net audiences are relatively small groups who have their own identity and "speak" the same language. Their language is marked by many new words especially coined to denote new phenomena that has emerged in the virtual medium. Such words place the members of the virtual community in certain subgroup e.g. wannabees - aspiring hackers who can't hack (Crystal 2004:82), lurkers people who access a chatgroup but don't contribute to the discussion (Crystal 2004:53). Some identify lurking with spying. Flamers are those who send aggressive messages related to a specific topic and directed at an individual recipient (Crystal 2004:55). Flaming is more likely to happen in chatgroups than in e-mails. Newbies are the newcomers who don't have sufficient skills in the Internet communication (Crystal 2004:53).

The introduction of even the above not numerous data shows that the interconnection of internet slang and slang in general is obvious. Considering the main characteristics of general slang we have come to understand that the given linguistic phenomenon is accepted and used by members of certain subculture or group. As far as internet language is concerned it cannot be understood apart the Internet medium. Being the language of a certain subculture Internet slang can be considered a new variety of slang and as any other variety of slang it has its unique characteristics that is accessible to the members of the virtual community.

\section{Notes:}

1. Mainly derived from the first letters of a phrase, acronyms are meant to make the phrase easier to say and remember.

2. Abbreviation (from brevis ("short") is strictly a shortening, but more particularly, an abbreviation is a letter or a group of letters, taken from a word or words, and employed to represent a certain notion in brief.

3. Emoticons are a form of commonly used in messages, in online, or in . An emoticon is a textual expression representing the face of a writer's mood or facial expression. The use of emoticons can be traced back to the when they were commonly used in casual/humorous writing.

4. This does not refer to e-mails as the e-mail language is totally different from that of the chatgroup discourse. 


\section{References:}

1. Bethany, K.D. and Lighter, J. (1978) Is Slang a Word for Linguists? // American Speech 53: 5-17.

2. Crystal, D. (2004) Language and the Internet. Cambridge: Cambridge University Press.

3. Eble, C. (1996) College Slang: In-group Language Among College Students. Chapel Hill: University of North Carolina Press.

4. Hotten, C.J. (2008) A Dictionary of Modern Slang, Cant and Vulgar Words. Michigan: University of Michigan.

5. Rhiengold, H. (1993) The Virtual Community: Homesteading on the Electronic Frontier. NewYork: Harper Collins.

6. $<$ http://www.Wikipedia.org $>$

7. $<$ http://www.netlingo.com>

8. <http://www.netlingo.com>

9. $<\mathrm{http}: / /$ www.wordreference.com $>$

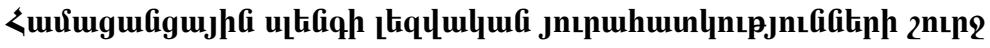

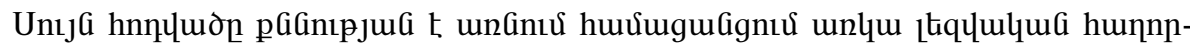

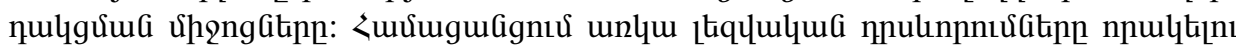

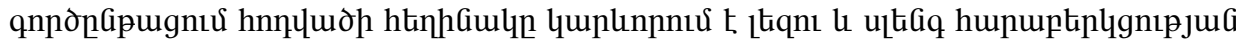

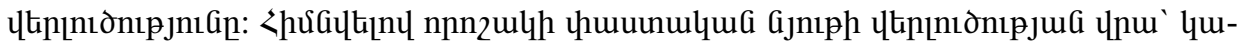

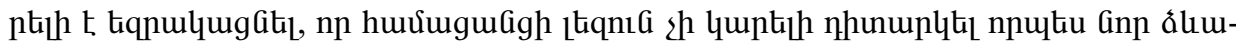

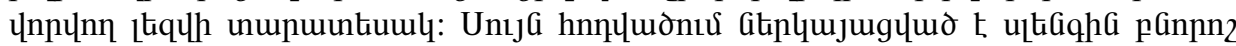

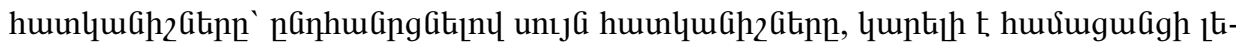

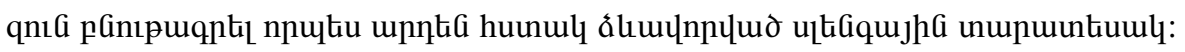

